\title{
Evolving role of molecular imaging with PET in detecting and characterizing heterogeneity of cancer tissue at the primary and metastatic sites, a plausible explanation for failed attempts to cure malignant disorders
}

\author{
Sandip Basu • Thomas C. Kwee • Robert Gatenby • \\ Babak Saboury • Drew A. Torigian • Abass Alavi
}

Published online: 31 March 2011

(C) Springer-Verlag 2011

\section{Introduction}

The diversity in cancer cell characteristics is an area of great interest in the practice of clinical oncology. By now, we know that tumors can be composed of different subpopulations of cells that can harbor very different characteristics (both in terms of genotype and phenotype), thereby exhibiting differential biologies including degrees of differentiation, growth rates, and response to various therapeutic interventions [1]. Intertumor heterogeneity across various malignancies and lesions (i.e., different biological characteristics between various lesions of the same malignancy or between different histological subtypes) has been relatively well described compared to intratumor heterogeneity (i.e., different biological characteristics within the subpopulations of neoplastic and non-neoplastic cells that successively evolve within a tumor mass) which, though recognized, has been

\section{S. Basu}

Radiation Medicine Centre (BARC),

Tata Memorial Hospital Annexe,

Parel, Bombay 400012, India

T. C. Kwee

Department of Radiology, University Medical Center Utrecht,

Utrecht, The Netherlands

\section{R. Gatenby}

Department of Radiology, Moffitt Cancer Center,

Tampa, FL, USA

B. Saboury $\cdot$ D. A. Torigian $\cdot$ A. Alavi $(\bowtie)$

Division of Nuclear Medicine, Department of Radiology,

University of Pennsylvania School of Medicine,

Hospital of the University of Pennsylvania,

3400 Spruce Street,

Philadelphia, PA 19104, USA

e-mail: abass.alavi@uphs.upenn.edu relatively less well investigated probably due to paucity of available techniques and methodologies. This regional intratumoral heterogeneity is manifested at the cellular level by multiple subpopulations with different genetic and phenotypic characteristics. Several newer tools (e.g., laser capture microdissection) have been utilized to study this phenomenon on a cellular basis in an apparently homogeneous tumor $[2,3]$. There is also tremendous temporal and spatial variation in the environment (and, therefore, environmental selection forces) that drive regional phenotypic evolution, largely as a result of variability of vascular density and blood flow. The prognostic significance of tumor heterogeneity has been emphasized in several malignancies, and in fact, grading systems have evolved in certain tumors based upon this characteristic [4-9]. To cite an example of its importance, in a study designed to determine genetic profiles of breast carcinoma cells, CD44+ cells had an active transforming growth factor beta (TGF-beta) pathway that correlated with decreased patient survival and poor prognosis compared to CD24+ cells [7]. It is important to note that heterogeneity is typically generated prior to clinical detection of the tumor, and that phenotypic diversity probably generally increases with tumor size, in parallel with the potential for genetic mutation to occur with each mitosis and the development of hypoxia, acidosis, ischemia, and necrosis.

Why cannot cancer be cured by a single treatment modality alone? Tumor heterogeneity, a plausible explanation

The most important practical utility of studying tumor heterogeneity lies in its implications for determining and defining therapeutic outcome in cancer. Recall that therapy 
fails for two general reasons: (1) phenotypic resistance, in which the cell is unresponsive to a treatment because of its intrinsic properties (such as activation of xenobiotic metabolism through overexpression of P-glycoprotein, upregulation of DNA repair pathways, etc.) and (2) de novo resistance, in which environmental factors such as hypoxia or ischemia reduce drug concentrations or the effectiveness of therapy in cells that would be otherwise sensitive.

It appears that even untreated tumors contain populations of resistant cells. This seems to arise as a result of cellular heterogeneity that allows cancer to broadly explore the available adaptive landscape. The Nowell hypothesis [10] and Goldie and Coldman hypothesis [11] are the two relatively well accepted theories of development of tumor heterogeneity in oncological parlance. The former states that despite having a common precursor cell, genetic instability allows for development of subsequent generations that have unique biological characteristics related to changes in both oncogenes and tumor suppressor genes. The latter states that the rate of development of tumor resistance is related to the size of the tumor: the larger the tumor, the greater the population of potentially drugresistant cancer cells, and hence the earlier it is detected the better the chance of therapeutic success. Both genetic and epigenetic phenomena, through upregulation and downregulation of certain genes, play an important role in the development of drug resistance. Of course, there is also a deep connection between the microenvironment and tumor phenotype, as changes in one invariably affect the other. Thus, regions of reduced vascularity and chaotic blood flow not only contribute to resistance through inadequate drug delivery but also through evolutionary selection forces that favor cells that are adapted to hypoxia and acidosis, leading to increased survival pathways and promotion of phenotypic resistance even prior to the onset of therapy. The role of environmental heterogeneity in promoting therapeutic resistance is of particular interest to the imaging community as clinical imaging can typically visualize and potentially quantify heterogeneity of tumor structure, blood flow, and metabolism. The question, then, is whether the observed heterogeneity that is apparent on imaging reflects the cellular and genetic variability that drives tumor biology and resistance to therapy.

\section{Tumor heterogeneity: a scale-invariant phenomenon}

We propose that the answer to the above question is "yes" and that the following is a fundamental principle of oncology: tumor heterogeneity is scale invariant. In other words, we hypothesize that there is consistent correlation between heterogeneity at a genetic and cellular level observed using molecular and microscopic techniques and heterogeneity of tissue level phenomena such as vascular density, blood flow, and regional metabolism at the molecular level, as measured by modern imaging. Thus, tumors that enhance uniformly on computed tomography (CT), for example, tend to have a regular pattern of cellular phenotypes on histological sections. In contrast, tumors that are environmentally heterogeneous with areas of variable enhancement and necrosis will also be highly variable at cellular and genetic levels. This hypothesis, which can be more formally stated as scale invariance of entropy or information, has been stated and explored mathematically [12-14]. However, for our purposes here, the main goal is to examine the related hypothesis that imaging, which can be used to readily measure tumor heterogeneity at a tissue scale, can also be used as a surrogate for heterogeneity at cellular and genetic scales. These observations, in turn, may provide insight into the prognosis of individual patients and predictions regarding the likelihood of response to therapy.

\section{Classification and clinical examples of tumor heterogeneity as revealed by positron emission tomography (PET)}

In vivo molecular imaging with PET can be regarded as a true classifier with regard to tumor cell characteristics as it can provide a global assessment of a given tumor via characterization of the subpopulations of the cell (Table 1). Through PET, tumor heterogeneity has clearly been observed in tumors in several scenarios. Prototypical examples that have been emphasized in the literature include NETs and other endocrine tumors (e.g., thyroid carcinoma). In these cancer types, clear disparities in tumor biology can be observed among different lesions (even within the same organ) through use of various PET radiotracers. For example, in the setting of thyroid cancer, some tumor lesions in a particular patient may have uptake of ${ }^{124} \mathrm{I}$ without uptake of FDG, whereas other tumor lesions may have no uptake of ${ }^{124} \mathrm{I}$ but have avid uptake of FDG [12-14]. This would mean that treating these patients with ${ }^{131} \mathrm{I}$ will fail in destroying the entire malignant cell population and eventually FDG-avid cells will determine prognosis and outcome in such settings. Before the introduction of FDG PET imaging, this flip-flop pattern was largely unknown; however, we now know that FDG-avid tumor volume plays an important role in determining patient prognosis $[15,16]$. Similarly, NETs are another subset of tumors that demonstrate this phenomenon of tumor heterogeneity. $\left[{ }^{68} \mathrm{Ga}\right] \mathrm{DOTA}$-octreotate PET imaging is significantly more sensitive for detecting small lesions than single photon emission computed tomography (SPECT) imaging and is now probably the most preferred agent for studying NETs. With the introduction of $\left[{ }^{68} \mathrm{Ga}\right]$ DOTA-octreotate PET/CT in 
Table 1 Spectrum of tumor heterogeneity observed in the parlance of clinical PET

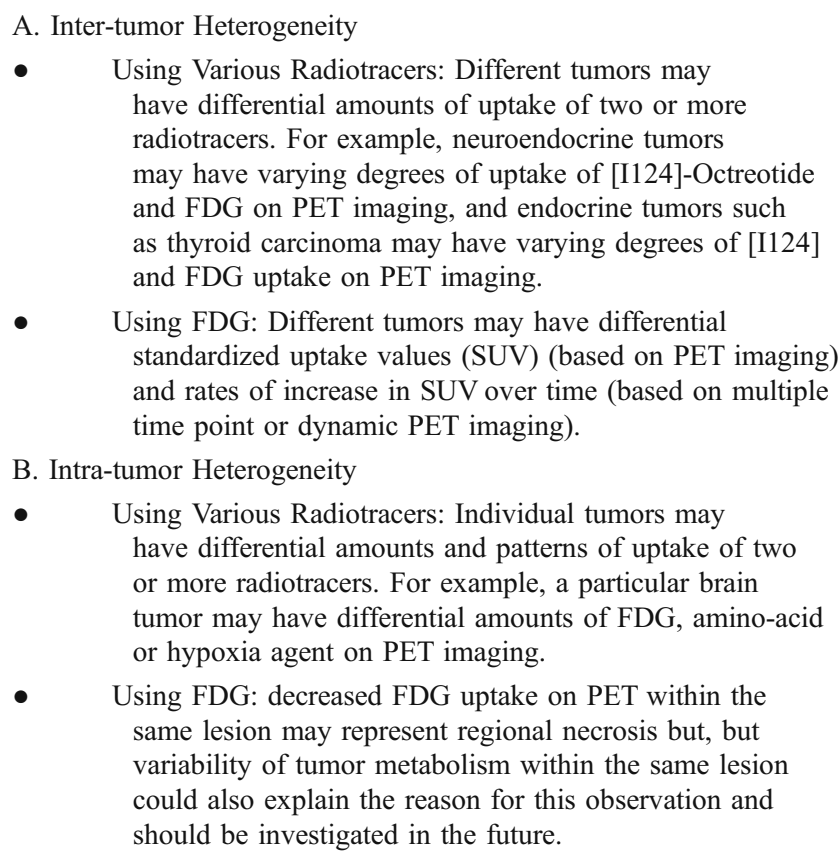

the routine clinical scenario, the phenomenon of diverse cancer characteristics in the functional imaging domain have been well documented. The loss of differentiation in this group of tumors often accompanies loss of somatostatin receptor (SSTR) expression (leading to reduced $\left[{ }^{68} \mathrm{Ga}\right]$ DOTA-TOC uptake) and an associated significant increase in glycolytic metabolism (leading to increased FDG uptake) [17-21]. Again, high FDG avidity in the tumors is usually associated with a worse patient prognosis $[22,23]$. Similar to what has been noted with thyroid cancer, it is not uncommon to visualize two or even three types of lesions in the same patient with NET where each group of abnormal tissues shows varying degrees of glycolysis, SSTR concentration, and dopaminergic metabolism.

On another note, FDG PET imaging has also provided us with another source of evidence in favor of this very important concept, where different tumors (and even different lesions in the same patient) behave completely differently with respect to the degree or rate of FDG uptake over time. It is not infrequent to observe that the rates of increase of FDG uptake in metastatic lesions in any particular anatomical location (e.g., the liver), let alone between different anatomical locations in any particular patient, may significantly vary. Furthermore, we have observed that the partial volume corrected SUV in primary lung cancer and in various metastatic sites may differ substantially, where bone marrow metastases had the highest SUVs compared to those in other organs [24].
These observations via PET imaging clearly demonstrate that tumor lesions in primary and metastatic sites may vary significantly with regard to their biology both among the various lesions in the same patient and within the same lesion. This suggests a major reason that many single-agent therapeutic interventions are unsuccessful to cure patients of their particular cancer.

\section{PET is the most robust in vivo imaging modality to depict intra- and intertumor heterogeneity in global fashion}

A reliable whole-body imaging modality that depicts tumor heterogeneity regionally and globally will play a crucial role to select and tailor various therapeutic regimens to individual patients with particular tumors. Modern structural imaging techniques, in spite of their major role in patient management, suffer from serious shortcomings with respect to assessment and quantification of tumor function and biology. These imaging modalities are unable to provide optimal characterization of malignant disorders and hence are likely to lead to ineffective therapeutic interventions. Even though molecular concentrations in tissues cannot be detected in humans beyond the picomolar level by utilizing PET imaging, currently, this modality appears to be the most robust methodology available for this purpose, outperforming existing structural (even other functional imaging techniques such as SPECT and NMR spectroscopy) imaging modalities in this setting. This is, in part, due to the ability to probe tumors in patients using a wide variety of radiotracers to explore various facets of intra- and interlesional heterogeneity in cancer. With the widespread availability of this imaging technique and the continuing evolution of novel PET radiotracers, this capability is only likely to expand further in the coming years.

\section{PET-depicted tumor heterogeneity as a critical determinant for predicting success of therapeutic response in cancer: implications for selecting and tailoring various treatment options}

We have already mentioned that PET imaging can demonstrate heterogeneity at a tissue scale between tumors from different patients and within a single tumor. Invoking our scale-invariant principle, we propose that tumors observed to be heterogeneous on imaging will be equally variable at cellular and molecular levels. This would indicate that the tumor response in a population of patients with radiologically variable tumors or in a single patient with radiologically heterogeneous tumors to a single therapeutic modality will be limited. This has two broad implications for treatment strategies. First, it is likely that no single therapeutic approach 
will be effective in a patient or population that exhibits tumor heterogeneity with modern imaging techniques. Second, it is unlikely that the current methods for individualized tumor therapy (such as microarrays or immunohistochemistry) will be predictive because the single parameter measurements obtained from these methods will be less accurate in heterogeneous tumor populations due to tissue sampling bias due to the limitations associated with this approach. In other words, we propose that selection of patient cohorts for specific targeted therapies should include both molecular and imaging characterization of their tumors. Similarly, any personalized treatment strategy must use imaging data to evaluate the accuracy and applicability of any molecular data set.

Thus, future combination chemotherapy regimens, consisting of multiple drugs, must be designed by taking into account the heterogeneity of tumor cells within the same patient. Likewise, the design of targeted radionuclide therapies in nuclear medicine must consider cellular and microenvironmental variability for improving probability of success with such treatments. For example, we know that $\left[{ }^{68} \mathrm{Ga}\right] \mathrm{DOTA}-$ octreotate PET imaging results form the basis for peptide receptor radionuclide therapy (PRRT) with for $\left[{ }^{177} \mathrm{Lu}\right]$ DOTAoctreotate in patients with NETs [25]. Yet, we also now know that increased glycolytic metabolism as demonstrated by FDG uptake is often associated with loss of SSTR expression (thus poor differentiation of malignant cells), and therefore will predict reduced efficacy of this treatment and overall poor prognosis and outcome. These patients, however, have an increased likelihood of response to chemotherapy. Similar observations have been also made while studying NETs of the sympathetic neuronal chain origin with metaiodobenzylguanidine (MIBG) imaging and deciding the suitability of a patient for $\left[{ }^{131} \mathrm{I}\right] \mathrm{MIBG}$ therapy [26-28].

We strongly believe that more PET assessment of tumor heterogeneity will have enormous implications for the individualized treatment of patients with cancer, and will be increasingly utilized on a routine basis in the future. It is likely that the development and success of new targeted therapeutics to overcome drug resistance in cancer will heavily rely upon PET by the academia and the industry in the coming years.

\section{References}

1. Marusyk A, Polyak K. Tumor heterogeneity: causes and consequences. Biochim Biophys Acta 2010;1805:105-17.

2. Johann DJ, Rodriguez-Canales J, Mukherjee S, Prieto DA, Hanson JC, Emmert-Buck M, et al. Approaching solid tumor heterogeneity on a cellular basis by tissue proteomics using laser capture microdissection and biological mass spectrometry. J Proteome Res 2009;8(5):2310-8.

3. Aubele M, Mattis A, Zitzelsberger H, Walch A, Kremer M, Hutzler P, et al. Intratumoral heterogeneity in breast carcinoma revealed by laser-microdissection and comparative genomic hybridization. Cancer Genet Cytogenet 1999;110(2):94-102.

4. Schilsky RL. Clinical implications of tumor heterogeneity. Haematol Blood Transfus 1987;31:278-82.

5. Krüger S, Thorns C, Böhle A, Feller AC. Prognostic significance of a grading system considering tumor heterogeneity in muscleinvasive urothelial carcinoma of the urinary bladder. Int Urol Nephrol 2003;35:169-73.

6. Hill RP, Chambers AF, Ling V, Harris JF. Dynamic heterogeneity: rapid generation of metastatic variants in mouse B16 melanoma cells. Science 1984;224:998-1001.

7. Shipitsin M, Campbell LL, Argani P, Weremowicz S, BloushtainQimron N, Yao J, et al. Molecular definition of breast tumor heterogeneity. Cancer Cell 2007;11(3):259-73.

8. Fidler IJ. Tumor heterogeneity and the biology of cancer invasion and metastasis. Cancer Res 1978;38(9):2651-60.

9. Wang N, Wilkin C, Böcking A, Tribukait B. Evaluation of tumor heterogeneity of prostate carcinoma by flow- and image DNA cytometry and histopathological grading. Anal Cell Pathol 2000;20(1):49-62.

10. Nowell P. Mechanisms of tumor progression. Cancer Res 1986;46:2203-7.

11. Goldie JH, Coldman AJ. The genetic origin of drug resistance in neoplasms: implications for systemic therapy. Cancer Res 1984;44:3643-53.

12. Gatenby RA, Frieden BR. Information theory in living systems, methods, applications, and challenges. Bull Math Biol 2007;69 (2):635-57.

13. Gatenby RA, Frieden BR. Application of information theory and extreme physical information to carcinogenesis. Cancer Res 2002;62(13):3675-84.

14. Frieden BR, Gatenby RA. Functional and malignant cell growth obey extreme information pathways (in press).

15. Wang W, Larson SM, Fazzari M, Tickoo SK, Kolbert K, Sgouros $\mathrm{G}$, et al. Prognostic value of $[18 \mathrm{~F}]$ fluorodeoxyglucose positron emission tomographic scanning in patients with thyroid cancer. J Clin Endocrinol Metab 2000;85:1107-13.

16. Robbins RJ, Wan Q, Grewal RK, Reibke R, Gonen M, Strauss HW, et al. Real-time prognosis for metastatic thyroid carcinoma based on 2-[18F]fluoro-2-deoxy-D-glucose-positron emission tomography scanning. J Clin Endocrinol Metab 2006;91:498-505.

17. Basu S, Kumar R, Rubello D, Fanti S, Alavi A. PET imaging in neuroendocrine tumors: current status and future prospects. Minerva Endocrinol 2008;33(3):257-75.

18. Frilling A, Sotiropoulos GC, Radtke A, Malago M, Bockisch A, Kuehl H, et al. The impact of $68 \mathrm{Ga}$-DOTATOC positron emission tomography/computed tomography on the multimodal management of patients with neuroendocrine tumors. Ann Surg 2010;252 (5):850-6.

19. Bhate K, Mok WY, Tran K, Khan S, Al-Nahhas A. Functional assessment in the multimodality imaging of pancreatic neuroendocrine tumours. Minerva Endocrinol 2010;35(1):17-25.

20. Naji M, Hodolic M, El-Refai S, Khan S, Marzola MC, Rubello D, et al. Endocrine tumors: the evolving role of positron emission tomography in diagnosis and management. J Endocrinol Invest 2010;33(1):54-60.

21. Kwekkeboom DJ, Kam BL, van Essen M, Teunissen JJ, van Eijck $\mathrm{CH}$, Valkema $\mathrm{R}$, et al. Somatostatin-receptor-based imaging and therapy of gastroenteropancreatic neuroendocrine tumors. Endocr Relat Cancer 2010;17(1):R53-73.

22. Binderup T, Knigge U, Loft A, Federspiel B, Kjaer A. 18Ffluorodeoxyglucose positron emission tomography predicts survival of patients with neuroendocrine tumors. Clin Cancer Res 2010;16:978-85.

23. Garin E, Le Jeune F, Devillers A, Cuggia M, de Lajarte-Thirouard AS, Bouriel C, et al. Predictive value of 18F-FDG PET and 
somatostatin receptor scintigraphy in patients with metastatic endocrine tumors. J Nucl Med 2009;50:858-64.

24. Bural G, Torigian DA, Houseni M, Basu S, Srinivas S, Alavi A. Tumor metabolism measured by partial volume corrected standardized uptake value varies considerably in primary and metastatic sites in patients with lung cancer. A new observation. Hell J Nucl Med 2009;12(3):218-22.

25. Ansquer C, Kraeber-Bodéré F, Chatal JF. Current status and perspectives in peptide receptor radiation therapy. Curr Pharm Des 2009; 15(21):2453-62.
26. Grünwald F, Ezziddin S. 131I-metaiodobenzylguanidine therapy of neuroblastoma and other neuroendocrine tumors. Semin Nucl Med 2010;40(2):153-63.

27. Postema EJ, McEwan AJ. Radioiodinated metaiodobenzylguanidine treatment of neuroendocrine tumors in adults. Cancer Biother Radiopharm 2009;24(5):519-25.

28. Basu S. Dose fractionation in 131I-metaiodobenzylguanidine (MIBG) therapy: should the tumour biology and intent of therapy be the guide? Eur J Nucl Med Mol Imaging 2010;37 (9):1798-9. 